\title{
A rare case of tumor-mimicking primary angiitis of the central nervous system
}

\author{
LI SUN $^{1 *}$, LIJUN ZHU ${ }^{2 *}$, TENG ZHAO $^{1}$, DAYAN WANG $^{3}$, DIHUI MA ${ }^{1}$, \\ RENSHENG ZHANG ${ }^{1}$ and SHAOKUAN FANG ${ }^{1}$ \\ ${ }^{1}$ Department of Neurology, The First Teaching Hospital of The Jilin University, Changchun, Jilin 130021; \\ ${ }^{2}$ Department of Neurology, The Third Teaching Hospital of The Jilin University, Changchun, Jilin 130033; \\ ${ }^{3}$ Qian Wei Hospital of Jilin Province, Changchun, Jilin 130031, P.R. China
}

Received September 4, 2015; Accepted February 4, 2016

DOI: $10.3892 / \mathrm{mco} .2016 .784$

\begin{abstract}
Primary angiitis of the central nervous system (PACNS) is a rare, but severe vascular disease. The present study reports the case of a 42-year-old male who developed PACNS. Magnetic resonance imaging (MRI) scans initially led to a misleading diagnosis of malignant glioma, and surgery was performed. The mass was resected, and a pathological examination confirmed a cerebral vasculitis. Single therapy with high doses of steroid did not improve the patient's condition, while a subsequent lesion appeared on the opposite side one year later. Combined therapy with methylprednisone and cyclophosphamide resulted in a great improvement for the patient. No relapse occurred during one year's follow-up. Although a tumor-mimicking PACNS has no established imaging features, a diagnosis of tumor-mimicking PACNS should be suspected when the MRI reveals inappropriate presentations of a tumor. Greater awareness of this potential manifestation of PACNS may facilitate more prompt diagnosis and treatment.
\end{abstract}

\section{Introduction}

PACNS is a rare and poorly understood form of vascular inflammatory disease, occurring in the brain and spinal cord. The average annual incidence of PACNS has been reported to be 2.4 cases per 1,000,000 person-years worldwide (1). The histopathological examination of biopsy specimens typically reveals that the arterioles and small

Correspondence to: Professor Shaokuan Fang or Dr Rensheng Zhang, Department of Neurology, The First Teaching Hospital of The Jilin University, 71 Xinmin Street, Changchun, Jilin 130021, P.R. China

E-mail: fang20063536@sina.com

E-mail: 415413119@qq.com

*Contributed equally

Key words: tumor-mimicking primary angiitis of the central nervous system, cyclophosphamide, malignant glioma arteries of the parenchyma and/or leptomeninges are affected by granulomatous inflammation (2). Non-specific clinical manifestations and variable imaging findings often lead to an incorrect or delayed diagnosis and treatment (3), particularly for the extremely rare form of tumor-like lesions. Furthermore, although a number of reports have described the characteristics of PACNS, no clinical case of PACNS in a patient from Northeastern China has previously been reported. In the present study, the clinical and radiological features of a case of histopathologically confirmed PACNS were retrospectively analyzed, in which PACNS was initially mistaken for malignant glioma.

\section{Case report}

A 42-year-old male patient was referred to the Department of Neurosurgery of our hospital due to a discontinuous headache and a 7-day history of deterioration, with 1 day of convulsions and aphasia. Magnetic resonance imaging (MRI) of the brain (using a Siemens Trio Tim 3.0T MRI scanner; Siemens AG, Munich, Germany) on admission revealed a tumor-like mass with edema, which was initially suspected to be a malignant glioma. On account of a clear shift of the midline, craniotomy was administered, and the lesion was totally resected (Fig. 1A), while pathological examinations revealed no identification of any tumoral texture by the surgeon. Concerned by a possible diagnosis of multiple sclerosis, a 3 day, high-dose $(1,000 \mathrm{mg}$ daily) pulse therapy of methylprednisolone was initiated. Serum and cerebrospinal fluid (CSF) immune routine examinations proved to be normal. Oligoclonal bands were negative. No marked increases in antiviral titers in the serum or the CSF were observed. One year later, the patient complained of seizures that had begun 2 months previously, and were characterized by 5 min episodes of twitching in the left leg that spread to his left arm. MRI of the brain revealed fluid attenuation inversion recovery ('FLAIR') hyperintensities in the right basal ganglia, and postoperative changes in the left frontotemporal lobe (Fig. 1B). With great interest, all the paraffin sections of the mass were carefully rechecked, and evidence for cerebral lymphocytic vasculitis, predominantly involving small-sized vessels, was eventually identified (Fig. 2). On the basis of the appearance of subsequent lesions in the right basal 


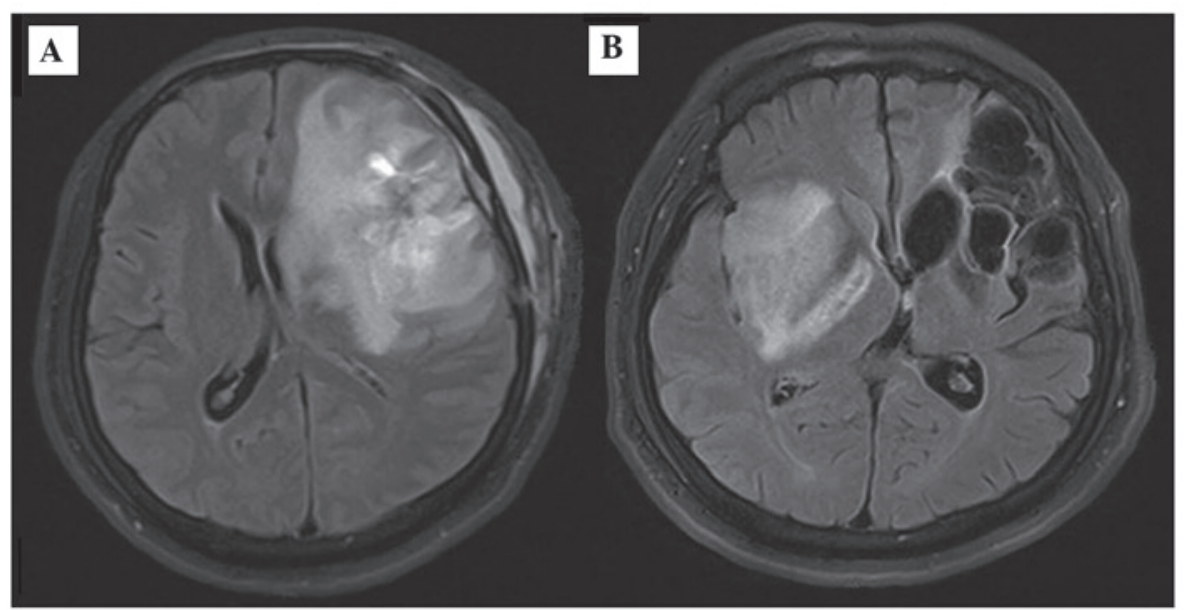

Figure 1. (A) Postoperative MRI scan showing flaky edema area in the left frontotemporal lobe (B) MRI fluid attenuation inversion recovery ('FLAIR') image showing the newly developed hyperintensities in the right basal ganglia and postoperative changes in the left frontotemporal lobe. MRI, magnetic resonance imaging.

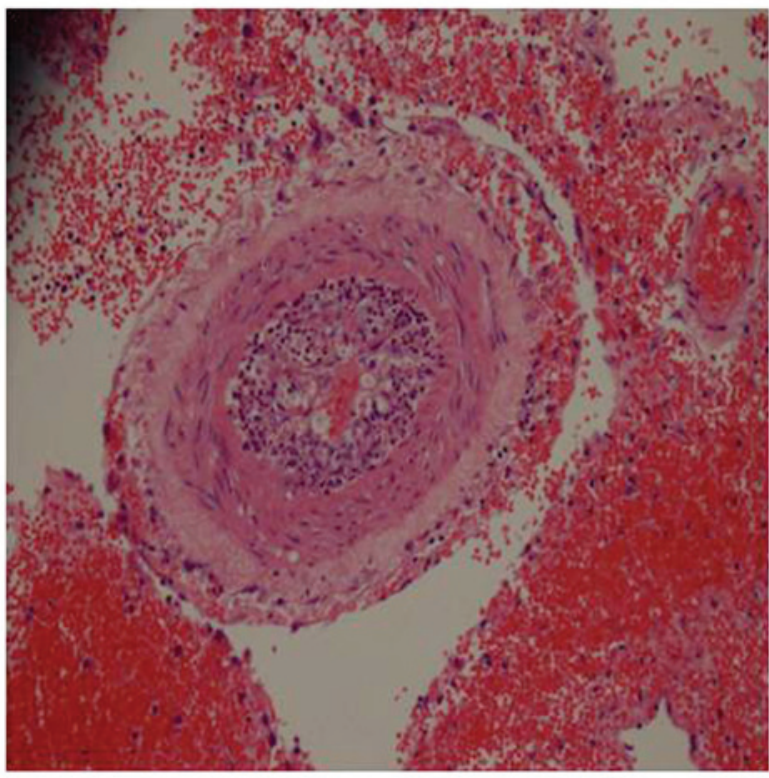

Figure 2. Hematoxylin and eosin-stained sections revealing lymphocytic inflammatory infiltrates in vessel walls and perivascular zones, focal fibrinoid necrosis in vascular walls and obliteration of parts of the vascular lumen.

ganglia, the patient was treated intravenously with methylprednisone at a dose of 1,000 mg/day for 3 days, $500 \mathrm{mg} /$ day for 3 days, $250 \mathrm{mg} /$ day for 3 days, and $125 \mathrm{mg} /$ day for 3 days, followed by oral prednisolone at $60 \mathrm{mg}$ /day and cyclophosphamide at $100 \mathrm{mg} /$ day for 2 weeks. At one year's follow-up, the patient had only aphasia, with no twitching in the limbs.

\section{Discussion}

To the best of our knowledge, this is the first case of clinically and pathologically confirmed PACNS in a patient from Northeastern China. Given that the prognosis of PACNS may be poor in the absence of specific therapy, early diagnosis and treatment is essential. Diagnosis is often delayed due to the extremely heterogeneous clinical presentations and non-specific
MRI features (4). PACNS presenting as a CNS mass lesion is extremely rare, and thus, PACNS is usually not considered in the differential diagnosis of mass lesions. A previous report indicated that only 3-5\% of the cases of PACNS presented as a CNS mass lesion (1). Furthermore, such cases are considered to be a fulminant form of focal CNS vasculitis, and only differ from typical cases in that they are more likely to be associated with amyloid angiopathy (1). Although usually non-specific, MRI features, including the involvement of grey and white matter, patchy contrast enhancement, intralesional hemorrhaging and normal choline with reduced $\mathrm{N}$-acetyl aspartate on magnetic resonance spectroscopy, may help to differentiate PACNS from other mass lesions caused by demyelination or neoplasms (5).

To date, biopsy has remained the gold standard for confirming the diagnosis of PACNS (6). Pathologically, PACNS can present as lymphocytic vasculitis, fibrinoid vasculitis or granulomatous vasculitis (7). The arteries affected are usually the smaller arteries and microscopic arterioles, and the presentation typically involves multiple arteries, and is multi-focal or multi-segmental. In the present case report, craniotomy was performed on the basis of a clear shift of the midline, whereas the pathological results predominantly revealed lymphocytic vasculitis. Whether this histological finding represents a specific characteristic of PACNS in Chinese patients remains to be elucidated, due to the small number of cases observed in the present study and the lack of published studies involving Chinese patients.

No clinical trials have been performed in patients with PACNS, and it is not possible to draw firm conclusions based on outcomes in the current case report due to the non-standardized nature of the treatment protocols used. Fountain et al (8) reported a case of PACNS controlled by administration of cyclophosphamide alone. Carandang and Grant (9) reported a female patient with PACNS who responded to steroid therapy alone. However, Barron et al (10) demonstrated that steroid therapy alone failed to improve the condition in their case study. Other reports have recommended combination therapy, consisting of prednisone and cyclophosphamide lasting at least 1 year (1). However, the patient in the present 
case study demonstrated a good response to treatment, combining steroid and immunosuppressant therapy. A high degree of suspicion, along with careful evaluation of the clinical, MRI and pathological data, is required to differentiate tumor-mimicking PACNS lesions from actual tumors and for a timely diagnosis and treatment. Early recognition is important, since tumor-like vasculitis usually responds poorly to steroid therapy. Furthermore, early combined therapy with aggressive immunosuppressive therapy and steroid may help to improve outcomes in patients with PACNS.

\section{References}

1. Salvarani C, Brown RD Jr, Calamia KT, Christianson TJ, Weigand SD, Miller DV, Giannini C, Meschia JF, Huston J III and Hunder GG: Primary central nervous system vasculitis: Analysis of 101 patients. Ann Neurol 62: 442-451, 2007.

2. MacLaren K, Gillespie J, Shrestha S, Neary D and Ballardie FW: Primary angiitis of the central nervous system: Emerging variants. QJM 98: 643-654, 2005.

3. Kraemer M and Berlit P: Primary central nervous system vasculitis: Clinical experiences with 21 new European cases. Rheumatol Int 31: 463-472, 2011
4. Molloy ES, Singhal AB and Calabrese LH: Tumour-like mass lesion: An under-recognised presentation of primary angiitis of the central nervous system. Ann Rheum Dis 67: 1732-1735, 2008.

5. Panchal NJ, Niku S and Imbesi SG: Lymphocytic vasculitis mimicking aggressive multifocal cerebral neoplasm: MR imaging and MR spectroscopic appearance. AJNR Am Neuroradiol 26: 642-645, 2005.

6. Kadkhodayan Y, Alreshaid A, Moran CJ, Cross DT III, Powers WJ and Derdeyn CP: Primary angiitis of the central nervous system at conventional angiography. Radiology 233: 878-882, 2004.

7. Koopman K, Uyttenboogaart M, Luijckx GJ, De Keyser J and Vroomen PC: Pitfalls in the diagnosis of reversible cerebral vasoconstriction syndrome and primary angiitis of the central nervous system. Eur Neurol 14: 1085-1087, 2007.

8. Fountain NB and Lopes MB: Control of primary angiitis of the CNS associated with cerebral amyloid angiopathy by cyclophosphamide alone. Neurology 52: 660-662, 1999.

9. Carandang CG and Grant AL: Delirium and isolated angiitis of the central nervous system: A case report and review. CNS Spectrums 13: 209-213, 2008.

10. Barron TF, Ostrov BE, Zimmerman RA and Packer RJ: Isolated angiitis of CNS: Treatment with pulse cyclophosphamide. Pediatr Neurol 9: 73-75, 1993. 